\title{
Consumo responsable, práctica de una ciudadanía ambiental
}

\author{
Diana Marcela Escobar Cabarcas \& María Camila Fontalvo Quintana \\ Facultad de Humanidades y Ciencias Sociales, Universidad del Norte, Barranquilla, Colombia. \\ dianaescobar021097@gmail.com,camila.maria950@gmail.com
}

Recibido: Noviembre 02, 2019.

Recibido en su versión corregida: Febrero 09, 2020.

Aceptación: Marzo 22, 2020.

Cómo citar: Escobar Cabarcas, D.M. \& Fontalvo Quintana, M.C, (2020). Consumo responsable, práctica de una ciudadanía ambiental. Revista Sextante, 22, pp. 25 - 32, 2020.

\section{Resumen}

El consumo responsable se basa en prácticas conscientes de consumo y elección de productos que llevan a un equilibrio en la cadena de consumo, manteniendo necesidades suplidas y un promedio aceptable de residuos sólidos. Las sociedades de consumo actuales reducen la promoción del consumo responsable por la rentabilidad económica que produce la obsolescencia programada, y aunque esto sigue siendo promovido por los ciudadanos, hay muchos que han ido implementando acciones proambientales que ayudan a reducir el impacto del mal consumo y sobre producción de los objetos. Con medidas educativas de cultura ciudadana y cultura proambiental se puede dar un giro al impacto medioambiental que estamos creando y seguir moviendo la economía.

Palabras clave: Ciudadanía proambiental; Consumo responsable; Cultura ciudadana; Obsolescencia programada.

\section{Responsible consumption, practice of environmental citizenship}

\begin{abstract}
Responsible consumption is based on specific consumption practices and choice of products that balance the consumption chain, the needs met and an acceptable average of solid waste. Current consumer societies reduce the promotion of consumption responsible for the economic profitability produced by planned obsolescence and although this continues to be promoted by citizens there are many who have been implementing pro-environmental actions that help reduce the impact of poor consumption and production of the objects. With educational measures of citizen culture and pro-environmental culture, the environmental impact we are creating can be turned and the economy moving.
\end{abstract}

Keywords: Civic culture; Pro-environmental citizenship; Responsible consumption; Scheduled obsolescence. 


\section{Introducción}

"El consumo responsable defiende los efectos positivos de un consumo cuidadoso con el medio ambiente y las personas, consciente, frente a un consumo excesivo, superfluo e innecesario, dañino para la vida del Planeta, y, por tanto, para todos sus habitantes." Ozonalia (Como se citó en Salas, 2009, p.24)

Teniendo en cuenta lo mencionado anteriormente es necesario realizar una mirada retrospectiva que relacione lo teórico con lo práctico, a fin de efectuar un análisis que permita desarrollar un bosquejo que conlleve a la autorreflexión desde las prácticas cotidianas responsables de un resultado que se hace inminente.

A lo largo del tiempo los problemas ambientales han tomado mayor relevancia, esto debido a las consecuencias que se originan a partir de éstas, las cuales son cada vez más tangibles, repercutiendo su impacto directamente en la sociedad. Como consecuencia de efectos adversos que se derivan de la inserción de la tecnología, desarrollo basado en el consumo y uso de los recursos naturales, se permite realizar múltiples reflexiones en cuanto al uso no responsable de los recursos, haciendo evidente el riesgo al cual estamos expuestos. Beck (como se citó en Ramírez, 2015). También es importante aclarar que según Arias (2016) la situación actual es alarmante por todo lo mencionado anteriormente y además porqué:

Refleja escenarios que no son los más propicios para asegurar la supervivencia humana $\mathrm{y}$, sin embargo, son muy pocos los que se detienen a pensar qué se puede hacer para revertir estos efectos nocivos, como es la contaminación en todas sus formas. Lo expuesto, conlleva a sugerir que la base de la problemática ambiental es producto del consumo en exceso de recursos. (p. 30).

A partir de lo anterior, es importante cuestionarse ¿Es Colombia ajena a la realidad de deterioro ambiental? la respuesta a este interrogante es no, a pesar de ser uno de los países con mayor biodiversidad, Andrade (como se citó en Ramírez, 2015) y uno de los países más ricos en cuanto a disponibilidad de agua OCDE (como se citó en Ramírez, 2015), Colombia afronta fuertes fases de deterioro ambiental que atañe a la calidad del ambiente. Ramírez (2015) menciona como una de las principales problemáticas ambientales en Colombia: la degradación de bosques y de suelo, deforestación, disminución de biodiversidad, sobreexplotación de recursos renovables, contaminación del agua y del aire, etc. Repercutiendo todos estos factores en la calidad de vida de las personas.

En un estudio realizado con estudiantes universitarios que se encontraban en 175 municipios de Colombia, según su percepción se logró identificar las problemáticas ambientales que más se percibían. Dentro de estas problemáticas 39,6\% mencionaron la contaminación del agua, 23,5\% la contaminación del aire y 18,9\% el mal manejo de residuos sólidos. Con la contaminación del agua se referían en mayor parte a la perturbación de la calidad del agua, ocasionada por elementos ajenos a su naturaleza; con respecto a la contaminación del aire, se hizo mayor énfasis en la emisión de gases por parte de vehículos e industrias manufactureras. Por último, en cuanto al mal manejo de residuos sólidos se mencionó el incorrecto manejo de residuos en espacios públicos (Ramírez, 2015).

Con el avance de la tecnología, la producción en masa y la revolución industrial, se desató todo un fenómeno en la producción de objetos. Este cambió el paradigma y se empezaron a crear nuevas necesidades en las personas para promover las compras. El problema surgió cuando grandes empresarios se plantearon la posibilidad de disminuir la vida útil de un producto para asegurar indefinidamente la compra del producto (Yang, 2016).

Hay dos estrategias que surgen a partir de esta necesidad de generar mayor consumo, la durabilidad artificial y la obsolescencia programada. En donde la primera se refiere al tiempo de vida útil que maneja un productor en la realización de su producto por la selección de productos de baja calidad. (Orbach, 2004) citado por (Yang, 2016). Por otro lado, en la obsolescencia habla de una vida útil programada, el producto es lanzado al mercado y tiene un tiempo estipulado de uso para que el consumidor deba comprar un nuevo modelo. La clave está en provocar esa necesidad y hacerle creer al consumidor que debe obtener esa 
nueva versión del producto para que la cadena siga siendo rentable para los productores. (Yang, 2016).

Toda la mecánica de consumo se crea con el fin de promover el consumismo que alimenta las políticas económicas de las empresas (Fernández, 2014). Las cuales están alineadas al funcionamiento económico de la sociedad. Crean cadenas de consumo cada vez más extensas y fuertes, en donde el consumidor crea la necesidad permanente de obtener los productos que van saliendo. De esta forma se mantiene la cadena de consumo que cada día, con el avance de las tecnologías, incrementa el nivel de residuos sólidos producidos. (Fernández, 2016).

Por lo tanto, para generar un cambio en la ciudadanía y en el mercado sobre un consumo más consciente y proambiental, se debe apuntar al cambio educativo y social, viéndolos como redes articuladas que deben trabajar juntas para generar el cambio transversal que se necesita. Pues lo social necesita de factores educativos bien establecidos para lograr una transformación y lo educativo necesita una sociedad activa y dispuesta. Por esto las estrategias pedagógicas dan el alcance de impactar desde pequeños grupos a grandes mayorías, sobre el impacto que tiene la decisión de comprar, consumo o utilizar algún producto o servicio, haciendo del consumo un acto más responsable. Esto por medio de la implementación de estrategias que incluyan todo el entorno de las personas, no solo reduciéndolo a un aula de clase, para así hacer partícipes a toda una comunidad y conectándolos con todos los recursos que los rodean. (Arias, 2016).

\section{Estado del arte}

En los años 20 se creó un fenómeno económico llamado obsolescencia programada que según Josep María Galí (2014) citado por (Niño, 2015), se materializa como "unos malos" los fabricantes y sus malévolos ingenieros y diseñadores industriales, que planean de forma consciente productos que se estropean tan rápidamente como lo permite el mercado para provocar nuevas ventas a través de innovaciones poco relevantes". (p.p 106)

Con el surgimiento de este fenómeno en la época de la Revolución Industrial, el mundo dio un giro centrándose en la producción y consumismo. (Niño, 2015). Se notó el consumo como la acción de adquirir bienes y servicios que satisfacían una necesidad no satisfecha. (Arias, 2016). Vemos cómo el mundo reinventó su forma de ver la vida, los productos que usaban y las prioridades, pues cada aparato que salía suplía una nueva necesidad, y en muchas ocasiones el mercado creaba esa necesidad para que ellos mismos la pudieran suplir. (Niño, 2015) ;(Báez-Mojica \& Cárdenas-Samudio, 2018).

Sobre el tipo de cultura capitalista y consumista que promovemos, se ha llegado a conclusiones como la del diseñador austriaco Víctor Papanek (1972) que dice que "la cultura occidental es una cultura kleenex, que desecha con pasmosa facilidad sus propias creaciones a merced del gusto cambiante" (Vargas, Vélez, Rodríguez \& Rojas, 2012:11) citado por (Niño, 2015). Lo que sirve de argumento para el "compro, luego existo" (BáezMojica \& Cárdenas-Samudio, 2018, p. 29) en el que el consumidor basa su existencia en el comprar y obtener bienes y servicios, y hace casi insatisfacible la saciedad de objetos o servicios. Nunca es suficiente, pues el mercado cada día encontrará la forma de renovarse y generar más necesidades.

Una gran consecuencia que ha generado tanto impacto positivo como negativo para el desarrollo ha sido el crecimiento tecnológico que se ha presentado en las últimas décadas (Fernández, 2014). Lo cual, en cierta forma, ha representado avances significativos que dan lugar al progreso o avance de distintos ámbitos, como a nivel social, económico, científico, etc. Sin embargo, en el manejo desmedido se encuentra lo que se considera contraproducente para el medio ambiente, esto es el incremento de basuras que deja este consumismo masivo. Residuos de una obsolescencia programada cada vez más corta. "Se estima que la producción anual de desechos electrónicos en todo el mundo ronda los 40 millones de toneladas" (Yang, 2016, pp 17). Todo esto por la falta de técnicas de reciclaje, de consumo responsable y otras dinámicas que mantengan la economía moviéndose, sin dejar cantidades industriales de residuos contaminantes. Por otro lado, adicionalmente a esto, como lo menciona Salas (2009) "desde que se originó la revolución informativa que dio paso a un mundo cada vez más globalizado, Colombia -como muchos otros países- comenzó a transformarse en un país cada vez más consumista” (p.44). 
Por esto, "el consumo tiene un lugar preponderante en la vida cotidiana y también en la crisis ambiental". (Mejía-Gil y Puerta, 2018, p. 79). "Esta crisis se refiere a una serie de problemas ambientales causados por actividades productivas industriales, que persisten a pesar de la revolución tecnológica" (CEPAL Naciones Unidas, 2016) citado por (Mejía-Gil \& Puerta, 2018, p. 78). Tanto así que se crearon en 2015, "los 17 Objetivos de Desarrollo Sostenible (ODS) y la agenda a 2030" (p.78).

Todo esto tiene un fin que mueve la dinámica del capitalismo, haciéndolo un modelo económico rentable $\mathrm{y}$ autosustentable, pues se crean las demandas y las ofertas, creando un círculo de necesidad. (Korstanje et al., 2008; Marinas, 2000; Veblen, 1974) citado por (Mejía-Gil \& Puerta, 2018); Báez-Mojica \& Cárdenas-Samudio, 2018). Como resultado de esto Salas (2009) menciona:

El colombiano por lo general busca consumir lo que su subconsciente acepta de la publicidad y no lo que su ser como tal le exige, o también consume porque el anuncio que vio fue muy convincente, pero al comprar dicho producto es muy dudoso que tenga en cuenta la responsabilidad social (p.10).

A fin de mitigar el resultado de lo anteriormente mencionado, se han buscado medidas que disminuyan el impacto de un consumo irresponsable y una producción desmedida. Por su parte, la ecología y el ambientalismo, "como movimientos sociales y campos científicos, comenzaron a promoverse después de la segunda guerra mundial, a partir de los primeros desastres ecológicos relacionados con el desarrollo económico de Europa y en EE. UU.” (Mejía-Gil \& Puerta, 2018, p. 78).

Lo anterior deja en evidencia la poca aceptación de un nuevo modelo que se venía implementando. Por esto, "la política de las instituciones internacionales busca impulsar el ambiente a partir de la redefinición de los patrones de producción y consumo", (Mejía-Gil \& Puerta, 2018, p. 79) lo cual requiere de:

un enfoque multidimensional e integral en el que la inclusión social, la sostenibilidad ambiental y el dinamismo económico se complementen. En la región, implica un profundo cambio de paradigma en las formas de operar del Estado, el mercado y la ciudadanía, así como el establecimiento de nuevas modalidades de colaboración entre ellos. (CEPAL Naciones Unidas, 2016, p. 173) Citado por (Mejía-Gil \& Puerta, 2018, p. 79).

Por otro lado, es importante destacar que hay nuevas generaciones de consumidores y prácticas de consumo en donde encontramos, según Elkington \& Hailes (1989; p. 235) citado por López \& Peñalosa (2018, p. 3), al que evita "productos que ponen en riesgo la salud del consumidor o de otro; aquellos que causan daño significativo al medioambiente durante la manufactura, su uso o desperdicio...". Esta práctica es mejor conocida como consumo verde, pero pronto tomó un camino más amplio al llegar el concepto de consumidor ético, por todas las cuestiones morales y éticas que estaban soportando las decisiones del consumo verde. Podemos limitar el consumo verde a la conciencia medioambiental del consumidor al momento de elegir un producto o servicio sobre otro. El consumidor ético, por otro lado, ve como un acto moral el proceso de compra o adquisición de productos o servicios. (Dueñas et al., 2014) citado por (López \& Peñalosa, 2018).

Hacer la distinción anterior es de suma importancia porque eso nos lleva a la evolución del mercado y consumo, en consciencia de actos que traen una consecuencia. Los conceptos anteriores se encuentran dentro uno más amplio, pues el consumo se ha ido expandiendo, ya no solo la toma de decisiones se basa en aspectos medioambientales o éticos, sino que también es importante la responsabilidad social de la empresa, el contexto de esta, aspectos generales que van más allá del simple producto o servicio, si no todos los procesos que están detrás de él. Concepto conocido como consumo socialmente responsable (CSR). (Mohr et al., 2001; Ozcaglar-Toulouse, 2005; Webb et al., 2008) citado por (López \& Peñalosa, 2018).

Con lo anterior denotamos el camino que el consumidor ha recorrido pues este ahora "tiene en cuenta las consecuencias públicas de su consumo privado e intenta usar su poder de compra para lograr el cambio social" (Webster, 1975) citado por (López \& Peñalosa, 2018.p, 4). Llevando el acto de compra al plano social, en donde priman los beneficios comunitarios antes que los individuales, 
es decir, que es más importante que los procesos que envuelven el servicio o producto manejen una estrecha relación proambiental, ética y social para luego poder adquirir cualquiera de estos. (Antil, 1984) citado por (López \& Peñalosa, 2018).

Sin embargo, es importante no dejar de lado las actitudes que deben estar presentes en los consumidores. Empecemos con el concepto de actitudes, que Schiffman y Kanuk (2010) explican: "una predisposición aprendida, que impulsa al individuo a comportarse de una manera consistentemente favorable o desfavorable en relación con un objeto determinado" (p. 228). Estas tienen 3 componentes Schiffman y Kanuk (2010):

El primero es el cognitivo, que es adquirido a través de la experiencia directa con el objeto y otras fuentes; el segundo es el componente afectivo, que determina las emociones o los sentimientos de un consumidor en relación con el objeto; y el tercero es el comportamental, que es el resultado de la conexidad entre los dos anteriores, con el cual un individuo realiza una acción específica en relación con la actitud frente al objeto. (228).

\section{Metodología}

Para la presente revisión bibliográfica se realizó una búsqueda relacionada con artículos asociados a problemáticas ambientales, consumo responsable, obsolescencia programada y ciudadanía proambiental.

Para la investigación se tuvo en cuenta artículos basados en datos científicos que permitieran soportar teóricamente la problemática a abordar en el presente escrito. Teniendo en cuenta el nivel de conocimiento de los autores consultados se logró contrastar varias nociones acerca la temática abordada con relación a definiciones de consumo responsable, conductas proambientales, obsolescencia programada, en conjunto con factores que permitían soportar explicación de fenómenos ambientales. También se permite entender a profundidad las razones que mueven el consumo, visto desde la producción, como desde parte del consumidor. Las investigaciones hacen un recorrido histórico extenso, demostrando cómo cada cambio en la tecnología y en la industria han marcado como vemos el consumo, llevándonos a la actualidad con sus peculiaridades cambios, que se espera lleven a la humanidad a mejorar la relación con el medio ambiente.

\section{Resultados}

Se hace evidente cuán inmerso se encuentra el ser humano en un entorno dotado de estímulos a los que se enfrenta diariamente $y$ ante los cuales debe asumir una postura responsable, bajo la toma de conciencia del impacto que generan las acciones tanto negativas como positivas.

Un consumidor responsable "Es una persona informada y consciente de sus hábitos de consumo. Además de conocer y exigir sus derechos como consumidor, busca la opción de consumo con el menor impacto negativo posible sobre el medio ambiente y con un efecto positivo en la sociedad. Esta manera responsable de consumir se traduce en muchos pequeños actos y decisiones diarias, y puede llegar a atravesar, todos los ámbitos de la vida (Salas, 2009, p.25).

Un consumidor responsable se caracteriza según Salas (2009) por adaptar su consumo a las necesidades justificadas, es decir, analizar si el objeto que se va adquirir es realmente necesario y por otro lado, es aquel que examina el impacto a nivel ambiental del objeto a adquirir, teniendo en cuenta el proceso productivo de este.

Son muchos los factores y consecuencias inmersas en un mal proceder en lo que se refiere al consumo responsable, dejando fuertes impactos tras generaciones. La Asociación de Profesores Amigos de la Ciencia (2007) menciona "Estamos, además, agotando recursos que van a repercutir sobre la vida de las generaciones futuras" (p.190). Frente a lo anterior es necesario tomar una postura basada en la autorreflexión que permita al ser humano analizar objetivamente sus acciones, siendo de este modo un ciudadano que haga una apuesta a lo ambiental desde sus actuar cotidiano. Pudiéndose gestar esta ciudadanía ambiental como lo menciona - () desde el entorno inmediato, donde se llevan a cabo las actividades cotidianas y expandiéndose a nivel global, relacionándose de esta manera un entorno con otro (Programa de ciudadanía ambiental global, 2005). 
Con el pasar del tiempo a través de jornadas ambientales, campañas e incluso desde la responsabilidad social empresarial de entidades que se han sumado a la causa de proteger el medio ambiente, se ha logrado dar a conocer el impacto de las acciones del ser humano sobre éste y así mismo, incitar la ejecución de conductas amigables con el entorno. Así como lo sustenta Salas (2009) cuando menciona:

La situación actual del medio ambiente, el elevado consumo de recursos naturales, la injusticia social, la gran brecha entre países pobres y ricos, la explotación, entre otras realidades son razones que hoy nos mueven a actuar de manera más consciente y justa (p.48).

\section{Discusión}

Teniendo en cuenta lo encontrado en las fuentes consultadas para la elaboración del presente artículo es evidente el impacto que tiene el consumo y manejo irresponsable de ciertos recursos para el medio ambiente y así mismo, para la calidad de vida humana. No proporcionando menos importancia a lo anterior, Arias (2016) se refiere a la falta de cultura ambiental como una de las causas básicas de las problemáticas de las cuales se ha estado hablando a lo largo del presente artículo, debido a que es por la irresponsabilidad ambiental ciudadana que se originan los altos índices de consumo, lo cual direcciona la relevancia que tiene la educación orientada hacia la promoción de sostenibilidad, a fin de brindar mayor calidad de vida siendo ciudadanos más conscientes de las propias acciones.

Como lo menciona Arias (2016) la educación es uno de los pilares más importantes de la actualidad, el cual sirve como base sólida para la formación de una sociedad comprometida con la sostenibilidad de las generaciones actuales y las futuras. Arias (2016) menciona que, si bien los consumidores actualmente están más enterados de sus hábitos de consumo, aún hay mucho en cuanto a factores negativos que se emanan de la relación ser humano - ambiente, razón por la cual es importante promover el análisis de las problemáticas que se están originando y que se derivan de falta de cultura. La educación proambiental debe ser soporte suficiente para contrarrestar los daños, permitiendo un cambio social.
En la misma línea, se tiene en cuenta la responsabilidad compartida que tienen tanto las empresas como el consumidor, al respecto Salas (2009) menciona:

En Colombia lastimosamente no está bien explotado el tema de comercio justo, ni consumo responsable, debido a la falta de conciencia por parte de la mayoría de las empresas y sobre todo del consumidor, quien ha optado por una posición conformista $\mathrm{y}$ consumista por encima de la satisfacción básica de sus necesidades y la falta de educación para exigir (p.10).

Independientemente si los autores hacen más énfasis en las empresas o consumidores como responsables del consumo desmedido, es necesario unificarlos en este proceso dónde están directamente relacionados con dicha problemática, impactando de manera significativa sobre el medio ambiente, esto debido a que como se mencionaba anteriormente, se crea una cadena donde se genera una necesidad que no es real, como resultado de estrategias que impulsan a la compra de productos o así mismo a la decisión poco regulada al momento de adquirir un artículo sin pensar en su proceso de elaboración y en el fin de su vida útil. En la misma línea, La Asociación de Profesores Amigos de la Ciencia (2007) mencionan "Hay que poner fin a la presión, guiada por la búsqueda de beneficios particulares a corto plazo, para estimular el consumo" (p.190).

Siguiendo lo anteriormente mencionado se hace necesario promover e incentivar la educación para la ciudadanía ambiental, a través de la cual se brinde la oportunidad de formar desde lo cotidiano, a fin de ser coherentes en las conductas emitidas que impactan directamente sobre el medio ambiente. Así lo menciona González (2003) "educar para la ciudadanía ambiental implica combatir contra una serie de elementos contradictorios que existen en el orden en que nos desempeñamos como sujetos sociales, que nos influye sobre cómo actuar en relación con el ambiente" (p.614).

De este modo, Pacheco (2006) menciona "Un concepto real de ciudadanía ambiental enfatizaría los derechos y obligaciones para con el medio ambiente, y considera la obligación de preservar los 
recursos naturales, cuidar de los ecosistemas y minimizar los impactos ambientales debidos a la contaminación” (p.156)

La participación ciudadana se vuelve imprescindible al momento de abordar temáticas relacionadas al medio ambiente, ya que de este modo es la misma ciudadanía quien brinda su opinión, aportando a la prevención y solución de problemáticas ambientales, lo cual hace que éstas sean sostenibles en el tiempo y se logren mejores resultados como producto de la adquisición de hábitos amigables con el medio ambiente (Montes y Valencia, 2012).

\section{Conclusión}

El consumo responsable trae consigo beneficios a nivel global con gran impacto positivo desde los distintos ámbitos de desarrollo, ya sea desde lo económico, social, científico, etc. Generado esto desde las acciones cotidianas del ser humano, que se originan por la toma de conciencia y educación ambiental, que además de repercutir sobre nuestro entorno, nos proporcionan bienestar propio.

Actualmente son muchas las explicaciones que surgen a fin de explicar las problemáticas ambientales que se están originando y que con el pasar del tiempo se hacen más evidentes. Algunos autores asumen el mal manejo de recursos como explicación a estos daños, otros un poco más profundamente basan su mirada desde la base de la problemática y se centran en la educación, mencionando la importancia de incentivar la reflexión en la sociedad a modo de contrarrestar esas implicaciones ambientales, que se están generando desde las acciones cotidianas del ser humano.

Resaltando los grandes resultados que en la actualidad podemos evidenciar, analizando, sobre todo, el momento de compra, la gente empieza a castigar a las marcas y de forma más social a los consumidores que tienen menos precauciones medioambientales y morales al momento de elegir. Hoy en día con el consumo verde, el consumidor ético y la importancia de la responsabilidad social de las empresas, el consumidor hace que el productor deba ser más cuidadoso en la extracción, producción y elaboración de productos, pues todo esto será importante al momento de adquirir bienes o servicios.

El consumidor ha optado por nuevos estilos de consumo que van más allá del impacto medioambiental y la responsabilidad social. Este también ha cambiado su estilo de vida, al preferir productos veganos, dietas más bajas en proteínas animales, con productos naturales. Miran mucho más cada ingrediente y el efecto que pueda ocasionar, tanto en el cuerpo, como en el medio ambiente.

Por todas las nuevas repercusiones que trae el consumo responsable, que cada día el consumidor conoce con mayor claridad, y las acciones premiadas por su entorno con un refuerzo positivo, vemos el aumento significativo en las conductas de consumo responsable en toda la sociedad. Además, las empresas han modificado sus procesos de extracción, manejo, producción y reproducción de los recursos, de forma que las repercusiones en el medio ambiente sean las mínimas.

\section{Referencias}

Arias, B. (2016). El consumo responsable: educar para la sostenibilidad ambiental. Aibi revista de investigación, administración e ingeniería, 4 (1),29-34

Asociación de Profesores Amigos de la ciencia. (2007). Consumo Responsable. Eureka, 4 (1), 189-192. Recuperado de https://www.redalyc.org/pdf/920/92040112.pdf

Báez-Mojica, C. E., \& Cárdenas-Samudio, N. (2018). Estrategia didáctica para fortalecer el pensamiento crítico en el tema de consumo responsable.

La ciudadanía ambiental global. (2005, septiembre). Recuperado de http://documentacion.ideam.gov.co/openbiblio/b virtual/019857/Ciudadaniaambientalglobal.pdf

Conteras, V \& Mejía, M. C. (2016). Contraconsumo: un análisis contracultural del veganismo, vegetarianismo y consumo responsable en la ciudad de Medellín. Universidad de Antioquia. http://bibliotecadigital.udea.edu.co/bitstream/10 
495/14759/1/ContrerasVladimir_2016_Contrac onsumoAnalisisContracultural.pdf

Fernández, L. (2014). La obsolescencia programada: sus consecuencias en el ambiente y la importancia del consumo responsable. UCES - Editorial de la Universidad de Ciencias Empresariales y Sociales. Terra Mundus. 2014, vol. $1, n .1$

González, E. (2003). Educación para la ciudadanía ambiental. Interciencia, 28(10), 611-615. Recuperado https://www.redalyc.org/pdf/339/33908509.pdf

López, D \& Peñalosa, M. (2018). El estereotipo de los colombianos frente al consumo socialmente responsable. Pensamiento \& Gestión, (44), $243-$ 260. https://dx.doi.org/10.14482/pege.44.10384

López, D. \& Peñalosa, M. (2018). Perspectiva de las actitudes de los Colombianos respecto al consumo socialmente responsable. Procceding. http://grupoinolsa.com/ojs3/index.php/proccedi ng_cladea2017/article/view/154/124

Mejía-Gil, M. C., \& Puerta, C. S. (2018). Consumo responsable y configuración de ciudadanías proambientales. Regions \& Cohesion, 8(1), 7777-106. doi: http://ezproxy.uninorte.edu.co:2084/10.3167/re co.2018.080105

Montes, J. y Valencia, F. (2012). Ciudadanía ambiental y agroecología Rutas para la sustentabilidad ambiental. Criterio Libre Jurídico, 9 (1), 109-117. Recuperado de file:///C:/Users/USER/Downloads/745-

Texto\%20del\%20art\%C3\%ADculo-1085-1-1020170829.pdf

Niño, L. (2015). Obsolescencia programada como fenómeno cultural. MasD, Revista Digital de Diseño. Vol. 9, Edición N. ${ }^{\circ} 16$ Ene. - Jun. 2015. 104-109

Pacheco, R. (2006). Ciudadanía ambiental global. Un recorte analítico para el estudio de la sociedad civil transnacional. Espiral, XII (35), 149-172.

Recuperado

de http://www.scielo.org.mx/pdflespiral/v12n35/v1 2n35a6.pdf
Ramirez, O. (2015). Identificación de problemáticas ambientales en Colombia a partir de la percepción social de estudiantes universitarios localizados en diferentes zonas del país. Revista Internacional de Contaminación Ambiental, 31 (3), 41-49.

Salas, C. (2009). El consumo responsable en Colombia (Tesis doctoral). Recuperado de https://javeriana.edu.co/biblos/tesis/economia/t esis206.pdf

Yang, Z. (2016). La obsolescencia programada. Universidad Del País Vasco, ADDDI. 